\title{
Evaluation of the Young's Modulus of Rubber-Like Materials Bonded to Rigid Surfaces with Respect to Poisson's Ratio
}

\author{
David Koblar1,* - Jan Škofic ${ }^{2}$ - Miha Boltežar ${ }^{3}$ \\ 1 Domel, Slovenia \\ 2 Iskra Mehanizmi, Slovenia \\ 3 University of Ljubljana, Faculty of Mechanical Engineering, Slovenia
}

Axially loaded rubber blocks with a circular cross-section, whose ends are bonded to rigid plates, were studied. The frequency-response functions were obtained with a finite-element model on rubber specimens with respect to Poisson's ratio and the shape factor. Then the apparent Young's modulus was estimated and various equations that describe the relationship between the apparent Young's modulus and the actual Young's modulus of the rubber material were used to estimate the Young's modulus of the rubber material. The subsequently estimated Young's modulus of the rubber material was compared to the one defined in the finite-element model.

It is shown that there is a significant difference in the estimated Young's modulus when different equations were used, especially when the Poisson's ratio of the rubber material is smaller than the theoretical value of 0.5 .

Keywords: Young's modulus, finite-element model, rubber, rubber-like

\section{INTRODUCTION}

Rubber materials are widely used for sound and vibration control and are usually bonded and compressed between rigid plates. In order to predict the vibration transferred through the rubber material in a finite-element model, knowledge of the material properties, such as the Young's modulus, the damping factor and the Poisson's ratio is essential.

An element of rubber material bonded to rigid plates, shown in Fig. 1, possesses an apparent Young's modulus $E_{a}$, which is due to the restrained motion of the upper and lower bonded surfaces, greater in value than the Young's modulus $E$ of the rubber material [1] to [4].

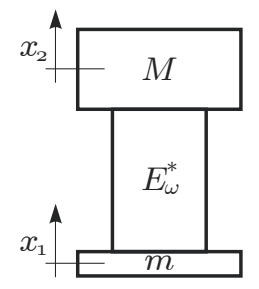

Fig. 1. Single-degree-of-freedom (SDOF) system, with ground excitation, measured displacement $x_{1}$ of the mass $M$ and the displacement $x_{2}$ of excitation

For a block with a circular cross-section Gent and Lindley [5] derived a correlation in the form:

$$
E_{a}=E\left(1+\beta S^{2}\right),
$$

where $S$ is the ratio of one loaded surface to the forcefree surface and $\beta$ is a numerical constant. For rubbers that are square, circular or moderately rectangular in cross-section $\beta=2$ should be used and for rubbers with the addition of carbon black, somewhat smaller values should be used [5].

For very large shape factors $S$ some contribution to the total deflection may be anticipated from the bulk compression of the rubber. To account for this, Gent and Lindley [5] derived the equation:

$$
\frac{1}{E_{a}}=\frac{1}{E\left(1+\beta S^{2}\right)}+\frac{1}{B}
$$

where $B$ is the Bulk modulus.

In deriving Eqs. (1) and (2) it was assumed that the material is virtually incompressible in terms of volume, that the cross-section of the block, normal to the direction of the applied load, remains plane and horizontal and also that the free vertical surfaces take up parabolic forms. Horton et al. [6] eliminated the assumption of a parabolic profile and derived a different expression:

$$
\frac{1}{E_{a}}=\frac{1}{E}\left(1+S \sqrt{\frac{2}{3}} \tanh \left(\frac{1}{S} \sqrt{\frac{2}{3}}\right)\right)+\frac{1}{B},
$$

where $B$ is again the Bulk modulus. Horton et al. [6] found that the results given with Eq. (3) are closely approximated by:

$$
\frac{1}{E_{a}}=\frac{1}{E\left(1.2+2 S^{2}\right)}+\frac{1}{B},
$$

and for a circular block of incompressible material, when $B=\infty$, can consequently be written as: 


$$
E_{a}=E\left(1.2+2 S^{2}\right)
$$

Williams and Gamonpilas [7] used the Timoshenko and Goodier [8] equilibrium equations and derived an expression in the form:

$$
\frac{E_{a}}{E}=\frac{1+3 v\left(\frac{1-v}{1+v}\right) S^{2}}{1+3 v(1-2 v) S^{2}} .
$$

Sim and Kim [9] developed a procedure to find a relationship between the ratio of the apparent Young's modulus to the Young's modulus, Poisson's ratio and shape factor. The downside of this method is that the transmissibility needs to be measured on two different specimens (one thin and one thick).

To run FEM calculations it is essential to know the actual Young's modulus. This is also important with other types of material models, see for instance [10].

In this paper a comparison of different equations for an estimation of the Young's modulus from an apparent Young's modulus derived from the transfer functions of a single-degree-of-freedom (SDOF) system will be presented. It will be shown that there is a significant difference in the estimated Young's modulus when different equations are used, especially when the Poisson's ratio of the rubber material is smaller than the theoretical value of 0.5 . The point of view presented above will be verified in this paper by using transfer functions obtained from a finite-element model of specimens having various shape factors and Poisson's ratios.

\section{THEORETICAL BACKGROUND}

The rubber is utilized so that its behavior is governed by the complex Young's modulus . Here it is assumed that the temperature remains constant with time, so that the complex Young's modulus may be written as [11]:

$$
E_{\omega}^{*}=E_{\omega}\left(1+\mathrm{i} \delta_{E_{\omega}}\right)
$$

where $E_{\omega}$ is the real part and $\delta_{E_{\omega}}$ is the ratio of the imaginary to the real part of the complex Young's modulus $E_{\omega}^{*}$, and is known as the damping factor and $\mathrm{i}$ is equal to $\sqrt{-1}$.

For a single-degree-of-freedom SDOF system, shown in Fig. 1, the transmissibility of the system, which is defined as the displacement ratio [11]:

$$
T=\frac{X_{2}^{*}}{X_{1}}=\frac{1+i \delta_{E_{\omega}}}{1-\omega^{2} \frac{h M}{A E_{a \omega}}+i \delta_{E_{\omega}}},
$$

and with real and imaginary parts of the transmissibility, $R E=\operatorname{Re}(T)$ and $\operatorname{IM}=\operatorname{Im}(T)$, known from the measurement, the frequency-dependent apparent Young's modulus can be obtained [11]:

$$
E_{a \omega}=\frac{h M \omega^{2}\left[I M^{2}+(R E-1) R E\right]}{A\left[I M^{2}+(R E-1)^{2}\right]} .
$$

Now the apparent Young's modulus needs to be converted to the Young's modulus of the rubber material. In the literature, [5] to [7], several equations were used. By transforming the Gent and Lindley [5], Eq. (1), frequency-dependent Young's modulus of the rubber material is derived:

$$
E_{\omega}=\frac{E_{a \omega}}{\left(1+\beta S^{2}\right)},
$$

or using Eq. (2) and taking the Bulk modulus:

$$
B=\frac{E}{3(1-2 v)},
$$

where $v$ is Poisson's ratio, into account indicates that the frequency-dependent Young's modulus of the rubber material is:

$$
E_{\omega}=E_{a \omega}\left(3+\frac{1}{1+2 S^{2}}-6 v\right) .
$$

Similarly, by rearranging Horton et al. [6], Eqs. (3) and (4), and taking into account the Bulk modulus, Eq. (11), the frequency-dependent Young's modulus of the rubber material is obtained:

$$
E_{\omega}=E_{a \omega} \frac{1}{3}\left(12-18 v-\sqrt{6} S \tanh \left(\frac{1}{S} \sqrt{\frac{2}{3}}\right)\right),
$$

and

$$
E_{\omega}=E_{a \omega}\left(3+\frac{1}{1.2+2 S^{2}}-6 v\right) .
$$

Next, the frequency-dependent Young's modulus is obtained by rearranging Horton et al. [6], Eq. (5), and is written in the form:

$$
E_{\omega}=\frac{E_{a \omega}}{1.2+2 S^{2}}
$$

The last equation is obtained by rearranging Williams and Gamonpilas [7], Eq. (6), where the frequency-dependent Young's modulus is expressed as: 


$$
E_{\omega}=E_{a \omega} \frac{(1+v)\left(-1+3 S^{2} v(-1+2 v)\right)}{-1+\left(-1+3 S^{2}(-1+v)\right) v}
$$

\section{ESTIMATION OF THE YOUNG'S MODULUS FROM THE FREQUENCY-RESPONSE FUNCION}

The frequency-response functions were obtained with a finite-element model in Ansys Workbench v14.5. The finite-element model represents a cuboid aluminum mass with a cross-section of $20 \times 20 \mathrm{~mm}$ and a height of $5 \mathrm{~mm}$, a cylindrical rubber with a diameter of $20 \mathrm{~mm}$ and different heights $(20,10,5$, 2.5 and $1.25 \mathrm{~mm}$ ), a cylindrical aluminum mass with a diameter of $50 \mathrm{~mm}$ and a height of $15 \mathrm{~mm}$, and an

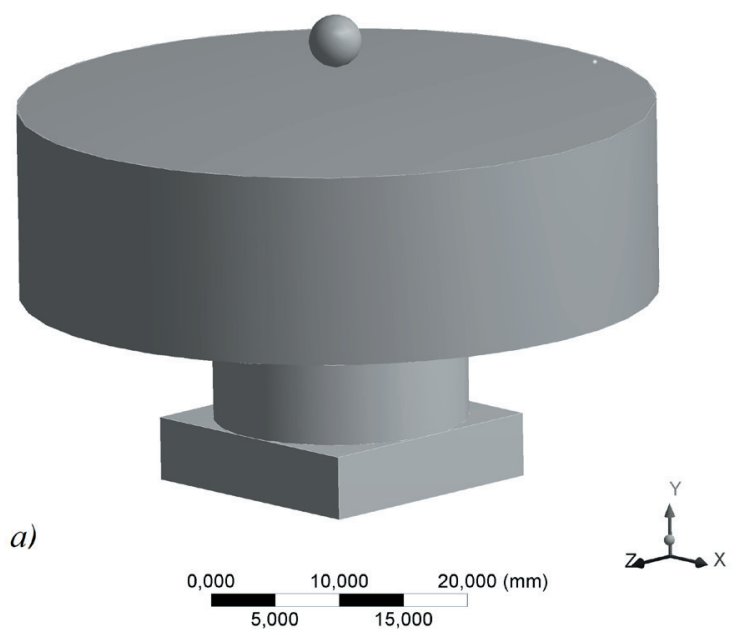

accelerometer on the top surface of the aluminum mass, which was modeled as a mass point of $4.6 \mathrm{~g}$, shown in Fig. 2a. The mesh was generated with a higher-order 3-D 20-node solid element that exhibits quadratic displacement behavior, SOLID186 and element MASS21 for the mass point. The generated mesh is shown in Fig. 2 b.

The material was assumed to behave as a linear elastic material with a constant Young's modulus of $4 \mathrm{MPa}$, defined in Ansys Engineering data, as was shown in [11] that the numerical calculations are in good agreement with the experimental measurements. Various Poisson's ratios, from almost incompressible $(v=0.4999)$ to compressible $(v=0.45,0.47$ and 0.49$)$ solids, were studied. Other material data are shown in Table 1.

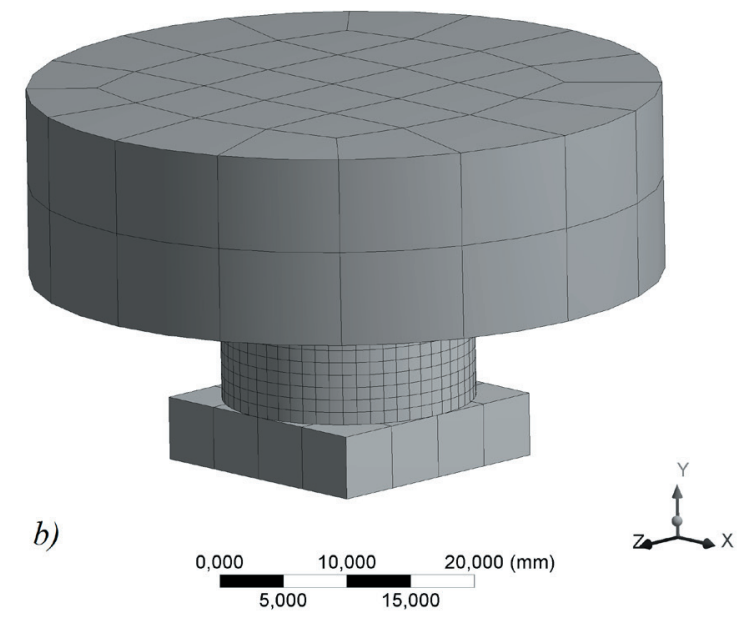

Fig. 2. Model in a) ANSYS Workbench and b) mesh

Table 1. Material parameters defined in ANSYS Workbench

\begin{tabular}{lcc}
\hline & Aluminum & Rubber \\
\hline density $\left[\mathrm{kg} / \mathrm{m}^{3}\right]$ & 2850 & 1200 \\
\hline Young's modulus [MPa] & $7.1 \times 10^{4}$ & 4 \\
\hline Poisson's ratio [-] & 0.33 & 0.4999 to 0.45 \\
\hline damping factor [-] & - & 0.01 \\
\hline
\end{tabular}

The harmonic analyses for combinations of five shape factors and four Poisson's ratios, in total 20 cases, were made in the frequency band from 20 to $5000 \mathrm{~Hz}$, with a frequency resolution of $1 \mathrm{~Hz}$. Then Eq. (9) was used to calculate the frequency-dependent, apparent Young's modulus and Eqs. (10) and (12) to (16) were used to calculate the values of the Young's modulus from the apparent Young's modulus. For the purpose of a clearer presentation the percentage error $E_{\text {error }}$ in the estimated values of the Young's modulus $E_{w}$ to the actual Young's modulus defined in the finite-element model $E_{\text {actual }}$ was calculated with the following equation:

$$
E_{\text {error }}=\frac{E_{\omega}-E_{\text {actual }}}{E_{\text {actual }}} \cdot 100 \%
$$

The percentage errors for various shape factors and depending on the value of Poisson's ratio are presented in Figs. 3 to 6.

\section{DISCUSSION}

Percentage errors in the estimation of the Young's modulus from the frequency-response functions calculated with the finite-element model, for $v=0.4999$, are presented in Fig. 3 and the percentage errors at $100 \mathrm{~Hz}$ are also shown in Table 2 . 


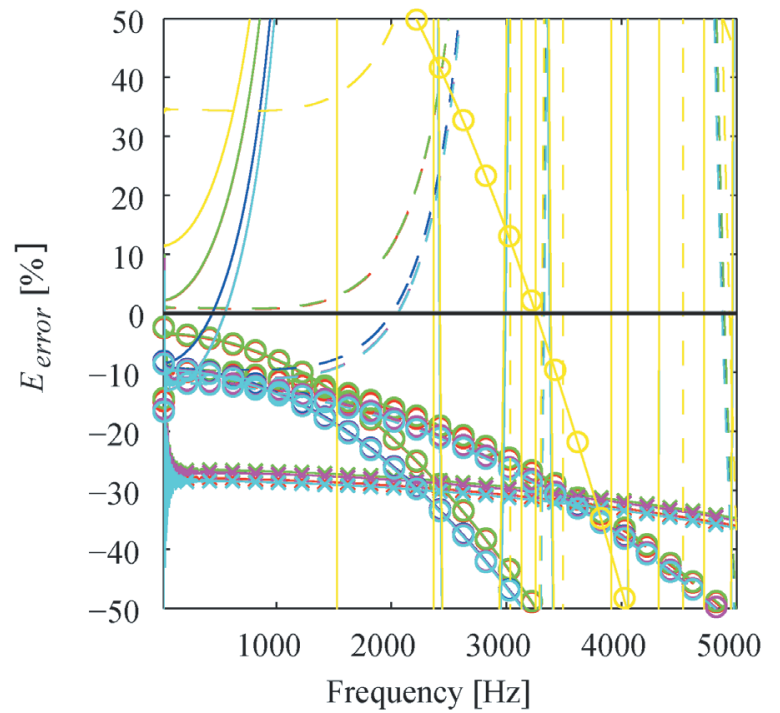

Fig. 3. Percentage errors of the estimated Young's modulus to the actual Young's modulus for different shape factors, (full) $S=0.25$, (dashed) $S=0.5$, (full with circle) $S=1$, (dashed with circle) $S=2$ and (full with $x) S=4$, and equations (red) Eq. 10, (green) Eq. 12, (blue) Eq. 13, (magenta) Eq. 14, (cyan) Eq. 15, (yellow) Eq. 16, at v $=0.4999$

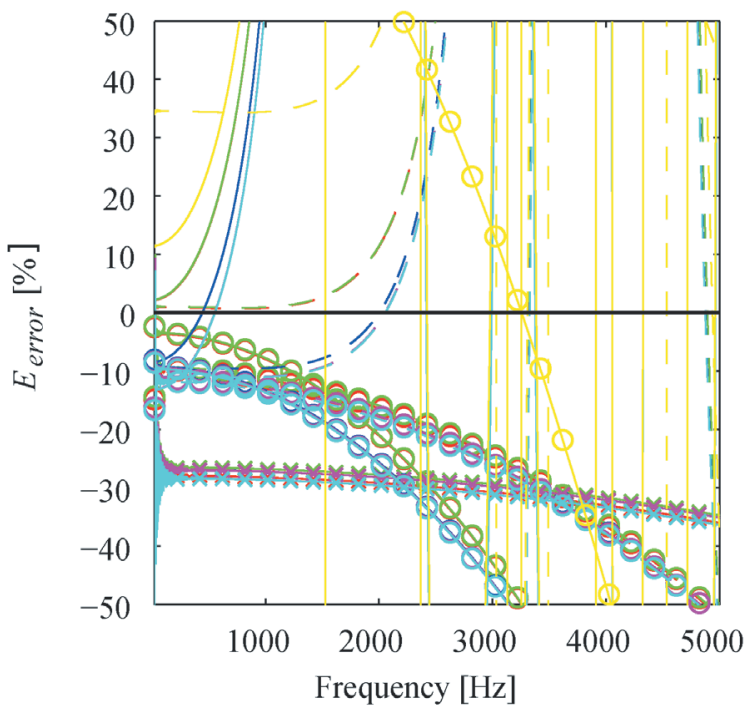

Fig. 4. Percentage errors of the estimated Young's modulus to the actual Young's modulus for different shape factors, (full) $S=0.25$, (dashed) $S=0.5$, (full with circle) $S=1$, (dashed with circle) $S=2$ and (full with $x) S=4$, and equations (red) Eq. 10, (green) Eq. 12, (blue) Eq. 13, (magenta) Eq. 14, (cyan) Eq. 15, (yellow) Eq. 16, at $v=0.49$

From this it can be concluded that Eqs. (10) and (12) give the best result when the shape factor is small (large thickness of the rubber) and the error increases with higher shape factors (with decreasing thickness of the rubber). It can also be seen that with a

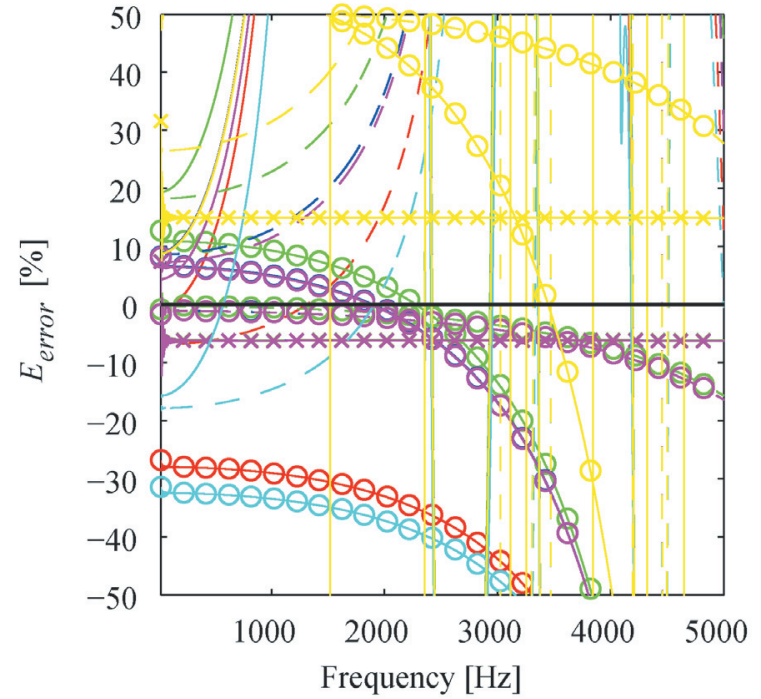

Fig. 5. Percentage errors of the estimated Young's modulus to the actual Young's modulus for different shape factors,

(full) $S=0.25$, (dashed) $S=0.5$, (full with circle) $S=1$,

(dashed with circle) $S=2$ and (full with x) $S=4$,

and equations (red) Eq. 10, (green) Eq. 12, (blue) Eq. 13,

(magenta) Eq. 14, (cyan) Eq. 15, (yellow) Eq. 16, at $v=0.47$

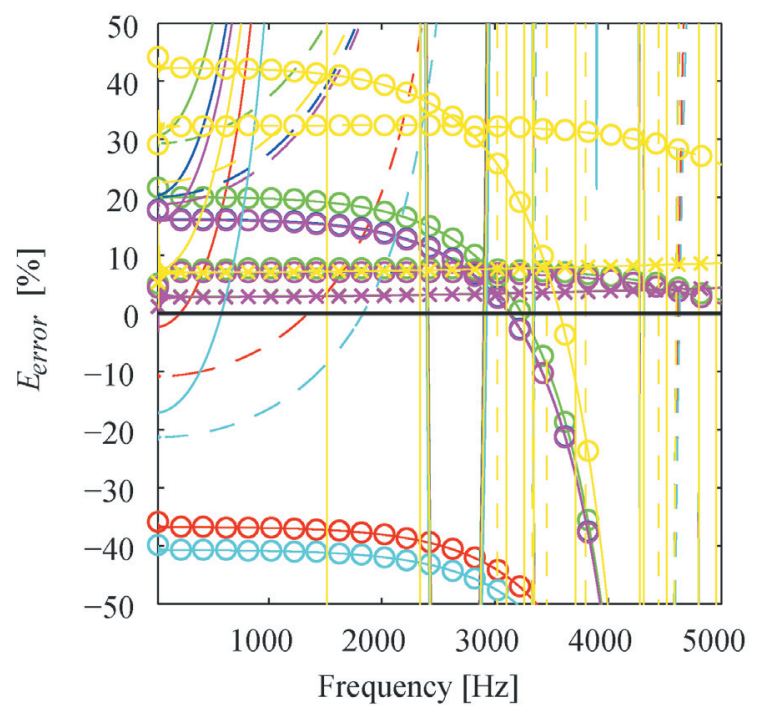

Fig. 6. Percentage errors of the estimated Young's modulus to the actual Young's modulus for different shape factors, (full) $S=0.25$, (dashed) $S=0.5$, (full with circle) $S=1$, (dashed with circle) $S=2$ and (full with x) $S=4$, and equations (red) Eq. 10, (green) Eq. 12, (blue) Eq. 13, (magenta) Eq. 14, (cyan) Eq. 15, (yellow) Eq. 16, at $v=0.45$

greater shape factor Eq. (12) gives better results than Eq. (10), since for very thin blocks some contribution to the total deflection may be anticipated from the bulk compression of the rubber, as discussed by Gent and Lindley [5]. 
Table 2. Percentage error in the estimation of the Young's modulus using different equations, at $100 \mathrm{~Hz}$ and $v=0.4999$

\begin{tabular}{cccccc}
\hline $\mathrm{h}$ [mm] & 20 & 10 & 5 & 2.5 & 1.25 \\
\hline $\mathrm{S}[-]$ & 0.25 & 0.5 & 1 & 2 & 4 \\
\hline Eq. (10) & 2.58 & 0.90 & -3.66 & -9.47 & -29.72 \\
\hline Eq. (12) & 2.65 & 0.99 & -3.48 & -8.98 & -28.33 \\
\hline Eq. (13) & -8.08 & -9.43 & -9.28 & -10.83 & -28.75 \\
\hline Eq. (14) & -12.83 & -10.88 & -9.5 & -10.95 & -28.75 \\
\hline Eq. (15) & -12.9 & -10.97 & -9.68 & -11.44 & -30.14 \\
\hline Eq. (16) & 11.91 & 34.54 & 92.74 & 171.91 & 158.93 \\
\hline
\end{tabular}

Estimated values of the Young's modulus with Horton et al.[6], Eq. (13), are underestimated by approximately $10 \%$, compared to the actual value of the Young's modulus defined in the finite-element model. Eqs. (14) and (15), which are approximations of Eq. (13), are very close together, where the maximum difference in the error compared to Eq. (13) is about $5 \%$ at $S=0.25$ and smaller than $1.6 \%$ at $S=0.5$. In the case of greater shape factors the difference is even smaller.

The difference in the estimation of the Young's modulus from Eqs. (10) and (12) to (15) is due to the approximation that with compression free vertical surfaces take up a parabolic form, Gent and Lindley [5], Eqs. (10) and (12), and that the profile of the compressed block is not quite parabolic, Horton et al. [6], Eq. (13) and its approximations Eqs. (14) and (15). Apparently, the Gent and Lindley [5] approximation of the parabolic profile, Eqs. (10) and (12), is the best fit for the calculations in Ansys. The maximum difference in percentage errors between Eqs. (10) and (12) to (15) for a small shape factor is $15.5 \%$ at $S=0.25$ and $12 \%$ at $S=0.5$ and at higher shape factors it is only $1.8 \%$ at $S=4$. From this it can be concluded that Eqs. (10) and (12) give the best results for all the addressed shape factors and Eq. (13), and its approximations Eqs. (14) and (15), give comparable results only for large shape factors (thin rubber).

The results given with Williams and Gamonpilas, Eq. (16), are not as expected. In the case of a small shape factor the percentage error is $11.9 \%$ at $S=0.25$ and is comparable to Eqs. (13) to (15), but in the case of a larger shape factor the Young's modulus is overestimated by $34.54 \%$ at $S=0.5$ and even $158.93 \%$ at $S=4$.

In the case that the rubber material is hardened by the addition of fillers, like carbon black, its Poisson's ratio is smaller than the theoretical 0.5. Consider that the Poisson's ratio is $v=0.49$. In this case the percentage errors in the estimation of the Young's modulus from the frequency-response functions calculated with finite-element models, are shown in Fig. 4 and the percentage errors at $100 \mathrm{~Hz}$ are also shown in Table 3.

It can be seen that Eqs. (10) and (15) do not take the Poisson's ratio into account and compared to the first case, this time error is greater than with Eqs. (12) to (14) that include the Poisson's ratio. From this it can be concluded that Eqs. (10) and (15) are not appropriate for an estimation of the Young's modulus when the shape factor $S \geq 1$. In this case Eq. (16) provides better results than in the case of incompressible rubber $(v=0.4999)$, but still does not come close to the results given with Eqs. (12) to (14). For Poisson's ratio $v=0.49$ Eq. (13) seems to offer the best performance in the estimation of the Young's modulus in the whole range of shape factors, followed by Eq. (14) and then Eq. (12).

Table 3. Percentage error in the estimation of the Young's modulus using different equations, at $100 \mathrm{~Hz}$ and $v=0.49$

\begin{tabular}{cccccc}
\hline $\mathrm{h}$ [mm] & 20 & 10 & 5 & 2.5 & 1.25 \\
\hline $\mathrm{s}[-]$ & 0.25 & 0.5 & 1 & 2 & 4 \\
\hline Eq. (10) & 1.45 & -2.14 & -14.26 & -39.85 & -72.19 \\
\hline Eq. (12) & 8.29 & 6.67 & 1.18 & -7.37 & -17.14 \\
\hline Eq. (13) & -2.32 & -3.44 & -3.98 & -8.66 & -17.30 \\
\hline Eq. (14) & -7.02 & -4.84 & -4.18 & -8.68 & -17.31 \\
\hline Eq. (15) & -13.87 & -13.65 & -19.62 & -41.16 & -72.36 \\
\hline Eq. (16) & 10.85 & 31.35 & 76.16 & 100.83 & 49.08 \\
\hline
\end{tabular}

In the case that the Poisson's ratio is $v=0.47$ or $v=0.45$, the percentage errors in the estimation of the Young's modulus from the frequency-response functions calculated with the finite-element model are shown in Fig. 5 and Fig. 6 respectively. It is clear that when the shape factor $S \geq 1$, Eqs. (10) and (15) are again not appropriate for an estimation of the Young's modulus. The estimation with Eq. (14) gives the best results and is followed by Eq. (13). Here, the results with Eq. (16) are improved compared to the rubber with the Poisson's ratio $v=0.4999$ and $v=0.49$ and for $S \leq 0.5$ are even better than the results given with Eqs. (12) to (14), but start to deviate for larger shape factors $S \geq 1$.

It should be noted that for small shape factors the Young's modulus can be estimated for a very narrow frequency band, e.g., for $S=0.25$ about $100 \mathrm{~Hz}$ and in the case of higher shape factors the Young's modulus can be estimated in a wider frequency band, e.g., for $S=0.5$ about $1000 \mathrm{~Hz}$ at $v=0.4999$ as shown in Fig. 3 . The upper limit of the frequency band is limited with wave effects that may develop at high frequencies of the transmitted vibrations as intense peaks resulting in 
a deterioration of the transmissibility, as reported by Rivin [12] and Snowdon [1], [13] and [14].

It is interesting that in the case $S \leq 0.5$ for all variants of Poisson's ratio Eq. (10) gives the best results and the percentage error is smaller than $10.8 \%$, which appears at $S=0.5$ and $v=0.45$ and even smaller in the case of $S=0.25$ where it is $2.6 \%$ at $v=0.4999$.

From this a general conclusion can be made, that for an estimation of the Young's modulus of rubber material to use with the finite-element analysis, it is possible to adapt the rubber dimensions to obtain a shape factor of around 0.5 and use the simplest Eq. (10) developed by Gent and Lindley [5]. With this approach it is possible to quite accurately estimate the Young's modulus of a rubber material, only the Poisson's ratio needs to be measured individually with a seperate procedure. Eq. (10) also gives the best results for all the shape factors if the rubber is almost incompressible, $v=0.4999$. Otherwise, if the shape factor $S \geq 1 \mathrm{Eq}$. (13) and its approximation Eq. (14) give the best result, but the Poisson's ratio of the rubber needs to be known in advance.

\section{CONCLUSIONS}

For an almost incompressible material $(v=0.4999)$, Gent and Lindley [5], Eqs. (10) and (12) offer the best accuracy for the actual Young's modulus, which was an input for the finite-element model, followed by Horton et al. [6], Eq. (13), with its approximations Eqs. (14) and (15).

In the case that the Poisson's ratio of the rubber material is not theoretical, $v=0.5$, for the shape factor $S \leq 0.5$ Eq. (10) quite accurately estimates the Young's modulus of the rubber material; however, as far as the shape factor $S \geq 1$, Eq. (13) and its approximation Eq. (14) give the best results.

With the proper selection of rubber dimensions, to have a shape factor of around 0.5 , it is possible to use the simplest equation for the estimation of the Young's modulus from the apparent Young's modulus, Eq. (10), and use the estimated Young's modulus for the finite-element analysis.

The downside is that the Poisson's ratio still needs to be measured individually, before it can be inputted into the appropriate equation.

\section{ACKNOWLEDGEMENTS}

Operation part financed by the European Union, European Social Fund.

\section{REFERENCES}

[1] Snowdon, J.C. (1968). Vibration and Shock in Damped Mechanical Systems, John Wiley and Sons, New York.

[2] Tsai, H.-C., Lee, C.-C. (1998). Compressive stiffness of elastic layers bonded between rigid plates. International Journal of Solids and Structures, vol. 35, no. 23, p. 3053-3069, DOI:10.1016/S0020-7683(97)00355-7.

[3] Koh, C.G., Lim, H.L. (2001). Analytical solution for compression stiffness of bonded rectangular layers. International Journal of Solids and Structures, vol. 38, no. 1, p. 445-455, DOI:10.1016/S0020-7683(00)00057-3.

[4] Tsai, H.-C. (2005). Compression analysis of rectangular elastic layers bonded between rigid plates. International Journal of Solids and Structures, vol. 42, no. 11-12, p. 3395-3410, DOI:10.1016/j.ijsolstr.2004.10.015.

[5] Gent, A.N., Lindley, P.B. (1959). The compression of bonded rubber blocks. Proceedings of the Institution of Mechanical Engineers, vol. 173, no. 1, p. 111-122, DOI:10.1243/PIME_PROC_1959_173_022_02.

[6] Horton, J.M., Tupholme, G.E., Gover, M.J.C. (2002). Axial loading of bonded rubber blocks. Journal of Applied Mechanics, vol. 69, no. 6, p. 836-843, DOI:10.1115/1.1507769.

[7] Williams, J.G., Gamonpilas, C. (2008). Using the simple compression test to determine young's modulus, Poisson's ratio and coulomb friction coefficient. International Journal of Solids and Structures, vol. 45, no. 16 , p. 4448-4459, DOI:10.1016/j. ijsolstr.2008.03.023.

[8] Timoshenko, S.P., Goodier, J.N. (1970). Theory of Elasticity, McGraw-Hill College, New York.

[9] Sim, S., Kim, K.-J. (1990). A method to determine the complex modulus and Poisson's ratio of viscoelastic materials from fem applications. Journal of Sound and Vibration, vol. 141, no. 1, p. 71-82, DOI:10.1016/0022460X(90)90513-Y.

[10] Mankovits, T., Szabó, T., Kocsis, I., Páczelt, I. (2014). Optimization of the shape of axi-symmetric rubber bumpers. Strojniški vestnik - Journal of Mechanical Engineering, vol. 60, no. 1, p. 61-71, DOI:10.5545/svjme.2013.1315.

[11] Koblar, D., Boltežar, M. (2013). Evaluation of the frequency-dependent Young's modulus and damping factor of rubber from experiment and their implementation in a FE analysis. Experimental Techniques, In Press, DOI:10.1111/ext.12066.

[12] Rivin, E. (2002). Vibration isolation theory. Braun, S.G. (ed.). Encyclopedia of Vibration. Academic Press, London, p. 1487-1506.

[13] Snowdon, J.C. (1979). Vibration isolation: Use and characterization. Journal of the Acoustical Society of America, vol. 66, no. 5, p. 1245-1274, DOI:10.1121/1.383546.

[14] Snowdon, J.C. (1958). The choice of resilient materials for anti-vibration mountings. British Journal of Applied Physics, vol. 9, no. 12, p. 461-469, DOI:10.1088/0508$3443 / 9 / 12 / 301$. 